\title{
Reversible Diffraction Efficiency of Photochromic Polymer Gratings Related to Photoinduced Dimensional Changes**
}

\author{
By Despina Fragouli, * Luana Persano, Giovanni Paladini, Dario Pisignano, Riccardo Carzino, \\ Francesca Pignatelli, Roberto Cingolani, and Athanassia Athanassiou
}

In this Full Paper, the possibility of reversibly changing the diffraction efficiency of gratings, fabricated by soft molding lithography on polymer films, containing photochromic molecules, is demonstrated. In particular, alternating UV and visible laser irradiation of the gratings causes the doped photochromic molecules to undergo transformations, which induce reversible dimensional changes to the samples. As a result, reversible changes are monitored in the intensity of the beams of a diode laser, transmitted and diffracted from the gratings. These changes affect the diffraction efficiency, which is increased upon irradiation with UV and decreased after irradiation with visible laser light. Such gratings are promising candidates for the fabrication of modern optical components such as optical switching devices.

\section{Introduction}

Holographic and diffractive optics attract great research and technological interest as they are incorporated in interconnection networks, ${ }^{[1]}$ light modulators, ${ }^{[2]}$ display devices, ${ }^{[3]}$ optical computers, ${ }^{[4]}$ information processing, and communication devices. ${ }^{[5,6]}$ Most of the research is focused on tunable gratings, namely, on nanostructured devices in which one can control the diffraction efficiency (DE) in a desired way. These can be based on photorefractive materials such as liquid crystals ${ }^{[7-10]}$ and polymers, ${ }^{[5,11]}$ on photochromic materials, ${ }^{[12,13]}$ or on their combinations. $^{[14-17]}$ The development of these gratings is generally done by interference of different polarized laser beams, or by electric-field application, and the modification of their DE is connected with the changes of their refractive index during this procedure.

In this work, we follow a different approach for the grating formation, and for the control of their diffraction efficiency, which relies on the dimensional variations of the gratings upon laser irradiation. In particular, the gratings are formed by the

[*] Dr. D. Fragouli, Dr. L. Persano, G. Paladini, Dr. D. Pisignano Prof. R. Cingolani, Dr. A. Athanassiou

National Nanotechnology Laboratory (NNL), INFM, CNR

Via Arnesano, 73100 Lecce (Italy)

E-mail: despina.fragouli@unile.it

Dr. D. Pisignano, R. Carzino, Dr. F. Pignatelli, Prof. R. Cingolani, Dr. A. Athanassiou

Italian Institute of Technology (IIT)

Via Morego 30, Genova (Italy)

[**] The authors would like to thank Dr. Marco Salerno, Dr. Francesco Rizzi, Dr. Monica Distaso, and Dr. Claudio Canale of the Italian Institute of Technology for their contribution to the experimental work. soft-molding technique, ${ }^{[18]}$ which presents the advantages of both soft and nanoimprint lithography, on a macromolecular matrix combined with photochromic spiropyran (SP) molecules. Using selective photon energy, reversible changes are caused to the system and are connected with the light-induced modifications to the active SP molecules. The colorless SP (Fig. 1I) undergoes a cleavage of its carbon-oxygen $\left(\mathrm{C}_{\text {spiro }}-\mathrm{O}\right)$ bond upon UV-light excitation, and subsequent isomerization to the colored open and polar form, merocyanine (MC; Fig. 1II). MC can revert back to the SP form photochemically, using visible-light irradiation. Thus, UV and visible irradiation cause the reversible transformation of these chemical species, between two states (isomers) that have light absorption bands in distinctive spectral regions. ${ }^{[19]}$ Moreover, other physical and chemical properties of these molecules, such as dipole moment, surface energy, refractive index, and volume, also change reversibly. ${ }^{[19]}$

The reversible response of the photochromic compounds to light exposure is proved to be retained when the photochromic molecules are incorporated in macromolecular matrices, where they are homogeneously dispersed forming miscible systems. ${ }^{[19-22]}$ In addition, in a previous study it was proved that the photochromic transformation between the SP and MC molecules can alter the macroscopic volume of their host polymer matrices reversibly upon light irradiation. In particular, reversible bending of a free-standing SP polymer film, caused by mechanical cycles of contraction/lengthening that are induced and controlled by UV-and green-laser pulses, was monitored. ${ }^{[19]}$ This property of the reversible dimensional changes is also observed in the gratings prepared in this work and is translated into changes of the intensity of the diffracted light. In this way we manage to produce switchable gratings that exhibit all-optically controlled changes of their DE, exploiting their structural alterations. The control of the DE 


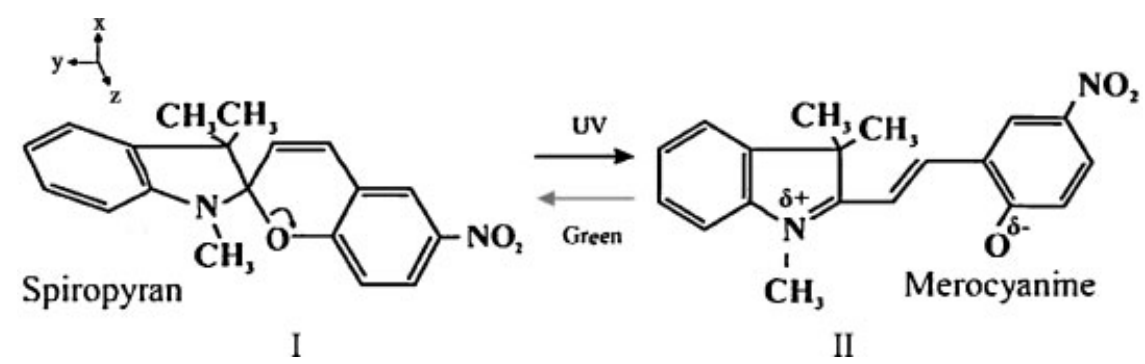

Figure 1. Scheme of the reversible transformation between the spiropyran (I) and merocyanine (II) isomeric forms induced by UV-visible irradiation.

needs only two laser beams of different wavelengths, is independent from the light polarization, and it requires a simple formation technology compared to other tunable DE devices..$^{[9,13,15]}$

a)
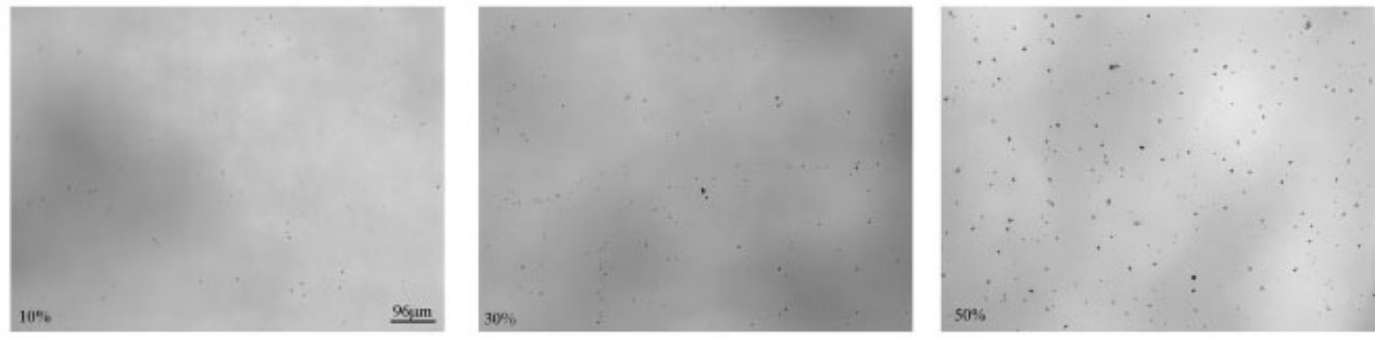

b)

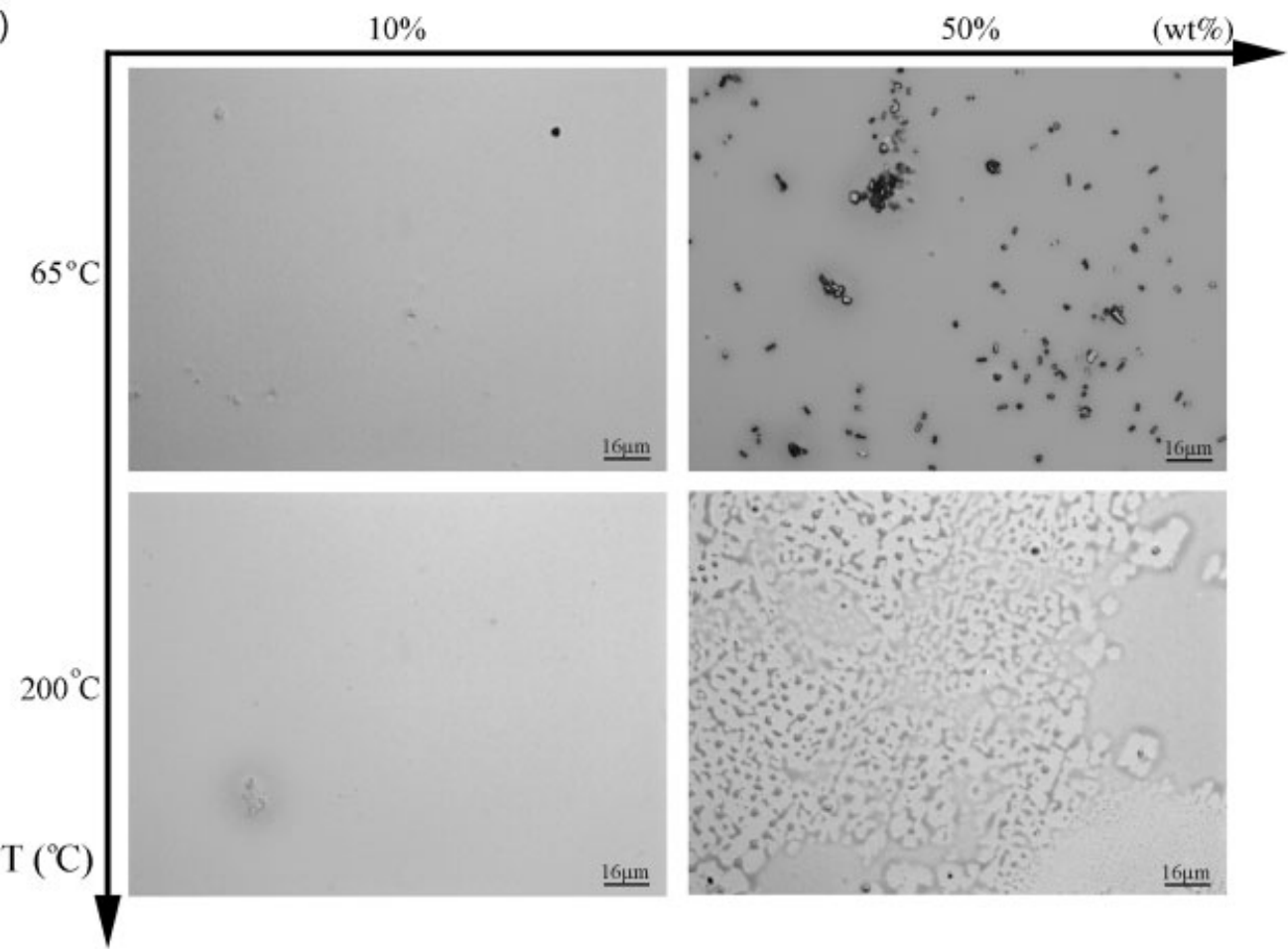

Figure 2. Optical micrographs of SP-PEMMA films at different relative concentrations and temperatures: a) at room temperature for three different concentrations of SP (from left to right: 10,30, and $50 \mathrm{wt} \%$ SP-PEMMA). The magnification is 10×. b) SP-PEMMA films taken after heating the samples at 65 and $200^{\circ} \mathrm{C}$, as indicated, showing a decrease in the homogeneity at high temperature and high concentration. The magnification is $50 \times$. 
temperatures. Figure 2a illustrates the film morphology of three representative concentrations, where it is clearly shown that the $10 \mathrm{wt} \%$ SP-PEMMA film is completely homogeneous and transparent; while for increasing SP concentration, the films exhibit some areas of possible aggregation. Next, the samples were heated at different temperatures. Figure $2 b$ presents indicative images, in which it is clear that the morphology of the film with $10 \mathrm{wt} \%$ SP remains unaffected by the temperature increase; however, this is not the case for the higher concentration of $50 \mathrm{wt} \% \mathrm{SP}$. At temperatures as high as $200{ }^{\circ} \mathrm{C}$ a change in the films morphology is already observed for the $30 \mathrm{wt} \%$ SP sample.

Differential scanning calorimetry (DSC) measurements of PEMMA samples with different concentrations of SP proved that the addition of a small amount of SP in the polymer matrix $(5,10,15$, and $20 \mathrm{wt} \%)$ does not affect the glass-transition temperature $\left(T_{\mathrm{g}}\right)$ of the polymer (measured value of $T_{\mathrm{g}}=$ $39^{\circ} \mathrm{C}$ ), while at higher concentrations, the increase of SP concentration causes the decrease of $T_{g}$ by $1-2{ }^{\circ} \mathrm{C}$ (for $30 \mathrm{wt} \%$ $\mathrm{SP}, T_{\mathrm{g}}=38^{\circ} \mathrm{C}$; for $\left.50 \mathrm{wt} \% \mathrm{SP}, T_{\mathrm{g}}=37^{\circ} \mathrm{C}\right)$. No sample showed a melting point in the measured range $\left(-30\right.$ to $\left.250{ }^{\circ} \mathrm{C}\right)$, independent of the SP concentration. Conclusively, the concentration of $10 \mathrm{wt} \% \mathrm{SP}$ in the polymer matrix does not affect the polymer properties and the morphology of the films. All the above findings prove that the samples of $10 \mathrm{wt} \% \mathrm{SP}$ in PEMMA that we used for the preparation of the gratings were homogeneous and miscible. ${ }^{[25]}$

Figure 3 illustrates the absorption spectra of a $10 \mathrm{wt} \%$ SP-PEMMA thin film recorded before and after UV-visible irradiation. Upon UV irradiation with a pulsed Nd:YAG (neodymium-doped yttrium aluminum garnet) laser operating at the third harmonic ( $\tau_{\text {pulse }} \approx 5 \mathrm{~ns}, \lambda_{\mathrm{UV}}=355 \mathrm{~nm}$, fluence, $F=20 \mathrm{~mJ} \mathrm{~cm}^{-2}$ ), the MC structure is formed, as indicated by the appearance of a new absorption band in the visible region of the spectrum (ca. $565 \mathrm{~nm}$ ). The intensity of the peak increases with the number of UV pulses until a plateau is reached after 20 pulses. The saturation of the intensity of the MC peak suggests that photoisomerization is completed and

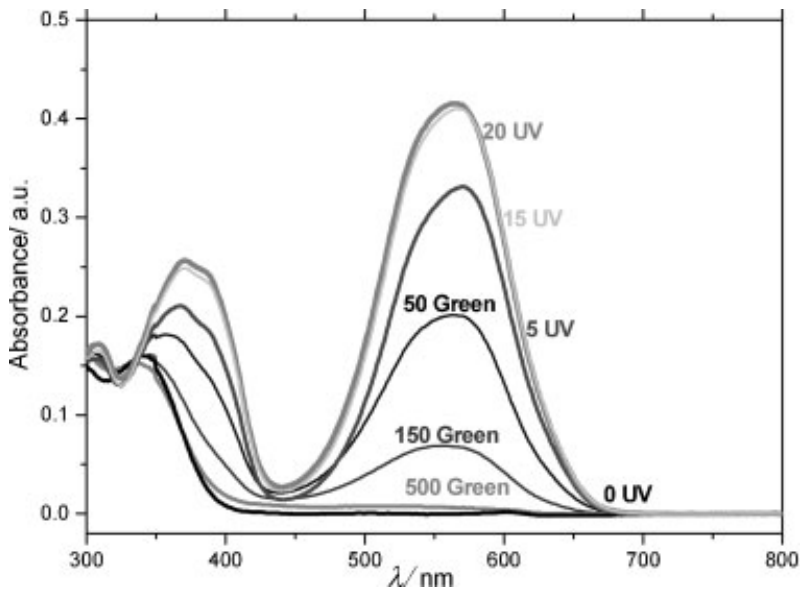

Figure 3. Absorption spectra of SP-PEMMA ( $10 \mathrm{wt} \% \mathrm{SP}$ ) substrates after irradiation with successive UV and visible irradiation. that the system has reached the equilibrium. After subsequent irradiation with visible light, produced by the second harmonic of the Nd:YAG laser $\left(\tau_{\text {pulse }} \approx 6 \mathrm{~ns}, \lambda_{\text {vis }}=532 \mathrm{~nm}\right.$, $F=35 \mathrm{~mJ} \mathrm{~cm}^{-2}$ ), the intensity of the previously formed peak decreases. After about 500 pulses, the spectrum again reaches its initial form, indicating that MC reverts to the SP structure. These data confirm that under the irradiation conditions used in this work the reversible properties of SP are retained in the host polymer matrix.

Using the above-mentioned irradiation conditions, the photoisomerization process is paralleled by a change of the first-order DE of the SP-PEMMA gratings with period of $4 \mu \mathrm{m}$ and thickness of approximately $240 \mathrm{~nm}$, formed by the softmolding technique, as described in the Experimental. All the gratings prepared for this study operate in the Raman-Nath (thin) regime. As shown in the inset of Figure 4, a continuouswave diode laser operating at $\lambda_{\text {laser }}=822 \mathrm{~nm}$ with a spot diameter of $1 \mathrm{~mm}$ is used as a reading beam. Its wavelength was carefully chosen so that the sample does not exhibit any absorption. The incident angle of the reading beam was adjusted to have the maximum intensity of the first diffracted line. The zero-order transmitted $\left(I_{0}\right)$ and the first-order diffracted $\left(I_{1}\right)$ lines of the reading beam, as measured by two photodiodes, were used for the calculation of the DE, as detailed in Equation 1. ${ }^{[10]}$

$\mathrm{DE}=\frac{I_{1}}{I_{0}}$

Measurements were conducted on five similar thin gratings, and Figure 4 shows the reversible first-order DE relative changes for one of them upon $3 \mathrm{UV}$-visible irradiation cycles. Each cycle presented in Figure 4 consists of $20 \mathrm{UV}$ pulses, $2 \mathrm{~min}$ in the dark, and 600 green pulses. The measurements

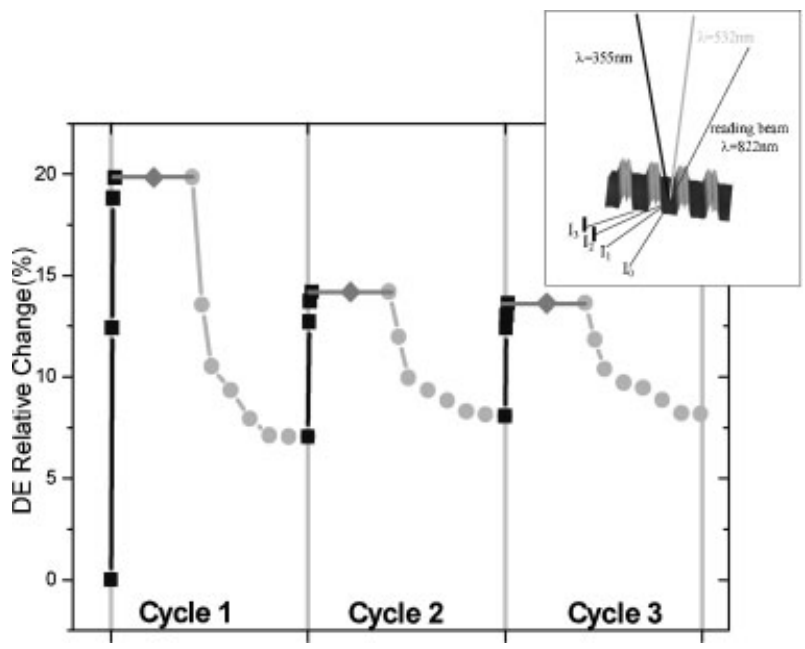

Figure 4. Relative changes in the diffraction efficiency (DE) of an imprinted grating during three irradiation cycles. Each cycle consists of 20 UV pulses, $2 \mathrm{~min}$ in the dark, and 600 green pulses. Inset: Optical setup for the generation and evaluation of the photoinduced changes of the DE of the gratings. 
were taken a few seconds after the end of each irradiation set to ensure complete relaxation of any thermal expansion of the samples. Indeed, previous studies made on polyurethane and poly(methyl methacrylate) (PMMA) showed that upon excitation at $248 \mathrm{~nm}$, at fluences below the corresponding ablation thresholds, thermal expansion/contraction occurs very quickly. Polyurethane recovers to the initial flat surface within $1 \mu \mathrm{s}$, and PMMA after approximately $5 \mu \mathrm{s} .{ }^{[26]}$ These times are much shorter than the few seconds left for the relaxation of the system before each measurement.

The initial DE of the presented grating was approximately $2.2 \%$. The DE increases upon irradiation with UV laser pulses and recovers close to its initial value after green pulses. The DE relative changes during the first cycle exhibit big variations between the various examined samples. This is mainly attributed to internal stresses of the polymer matrix, produced during the preparation of the gratings, that are released in a random way upon irradiation. ${ }^{[27]}$ During the following cycles the DE relative changes are similar for all the examined gratings. In particular, at the second cycle during UV irradiation the DE increases with increasing number of pulses, until it stabilizes to an average value of approximately $7.4 \pm 3.0 \%$ higher than the initial one, after 20 pulses. Subsequently, the green pulses cause the the DE to decrease, until stabilization to a value close to the initial one is obtained. This phenomenon is also observed in the third cycle and continues for at least seven cycles, with a decreasing trend, because of the photo-oxidation of the SP molecules close to the surface. ${ }^{[28]}$ It is worth noticing that the DE can remain stable for a few days if the grating is stored in the dark and it is further prolonged if the storage temperature is about $5^{\circ} \mathrm{C}$.

The reversible changes of the DE are attributed to the reversible light-induced macroscopic deformations of the nanoimprinted gratings. In particular upon UV irradiation, formation of aggregates between different MC stereoisomers with zwitterionic character occurs, causing density fluctuations in the polymer matrix. In order to these reduce density fluctuations, short-scale motion of the polymer chains may occur, causing the macroscopic contraction of the grating stripes. Next, upon green irradiation, the formation of the aggregates is less favored until it stops, caused by the return of the MC molecules to the SP form, which does not form aggregates. This mechanism is analytically described in a previous work by Athanassiou et al. ${ }^{[19]}$ Atomic force microscopy (AFM) measurements clearly show the reversible macroscopic changes of the gratings following the UV-green irradiation. Figure 5 presents the AFM images of one grating at the second irradiation cycle, before, after UV, and after green irradiation. Particularly, UV irradiation induces the decrease of the width of the stripes by $13 \%$ and the subsequent increase of the distance between the two stripes. A small decrease is also observed in the period of the grating, whereas after irradiation with visible light the values recover to very close to the initial one.

In the AFM images of some of the prepared gratings, as shown in Figure 5, we observe a dip separating each stripe in two equal parts. Our soft-imprinting technique mainly relies on the capillarity that allows the viscous polymer to spontaneously fill the vertical channels that are made of the recessed features of elastomeric elements, given that wetting lowers the overall free energy. As a consequence, because of the possibility of non-complete filling of such recessed regions by the photochromic blend, double-crest features can be obtained in the imprinted polymers, initially piling up in the regions adjacent to the protruding areas of the mold and forming dips in the central part of the growing capillarity features. This fluidic behavior is common to different imprint lithography methods, including hot embossing, ${ }^{[29]}$ room-temperature nanoimprinting, ${ }^{[30]}$ and soft molding. ${ }^{[18]}$ This dip is useful for the morphological analysis of the patterned surfaces, since it makes the demonstration of the volume changes upon alternating UV and visible irradiation by AFM much clearer. On the other hand, it is narrow enough to give any contribution to the diffracted light from the grating (Fig. 5).

After ellipsometric measurements on a $10 \mathrm{wt} \%$ SP-PEMMA sample, we found a refractive index of $n_{\text {PEMMA-SP }}=1.509$ for $\lambda=822 \mathrm{~nm}$. After UV irradiation, the refractive index is increased and the change is about $\Delta n=0.029$. This finding is very close to the value measured by Sasaki et al., ${ }^{[31]}$ who used a polystyrene film containing $10 \mathrm{wt} \% \mathrm{SP}$ and after the photoisomerization of the SP to MC they found an increase of the refractive index of approximately 0.02 at $\lambda=800 \mathrm{~nm}$. As this difference is very small compared to the periodic refractiveindex variations in the grating between the photochromic polymer (1.509) and the air (1), which is actually what causes diffraction to occur, we assume that it plays a negligible role in the relative changes of the DE. Furthermore, the small thickness of our samples $(\leq 250 \mathrm{~nm})$ reduces the importance of the refractive-index difference between the two isomeric states even more.

Using the basic equation that describes the intensity distribution of monochromatic light passing through a grating, we compare the experimental results with the theory (Eq. 2). ${ }^{[32]}$

$\frac{I}{I_{0}}=\left[\frac{\sin (\pi \beta p / \lambda)}{\pi \beta p / \lambda}\right]^{2}\left[\frac{\sin (N \pi \alpha p / \lambda)}{\sin (\pi \alpha p / \lambda)}\right]^{2}$

Specifically, $I$ and $I_{0}$ are the intensities of the light after the grating at various orders of diffraction and at zero order, respectively, $\beta$ is the distance between two successive stripes, $\alpha$ is the period of the grating, $N$ is the number of stripes, $\lambda$ is the wavelength of the reading beam, and $p=\sin \theta-\sin \theta_{0}=m \lambda / \alpha$ ( $m=0, \pm 1, \pm 2$, etc.), where $\theta_{0}$ is the angle of incidence and $\theta$ the angle of diffraction. The number of the stripes covered by the reading beam was calculated by dividing the diameter of the spot of the beam by the period of the grating in each case. The angle of incidence of the reading beam was $\theta_{0}=20^{\circ}$. From the above-mentioned values and Equation 2, and by taking into account the different values of $\alpha$ and $\beta$ measured from the AFM images before and after irradiation, we calculated the 


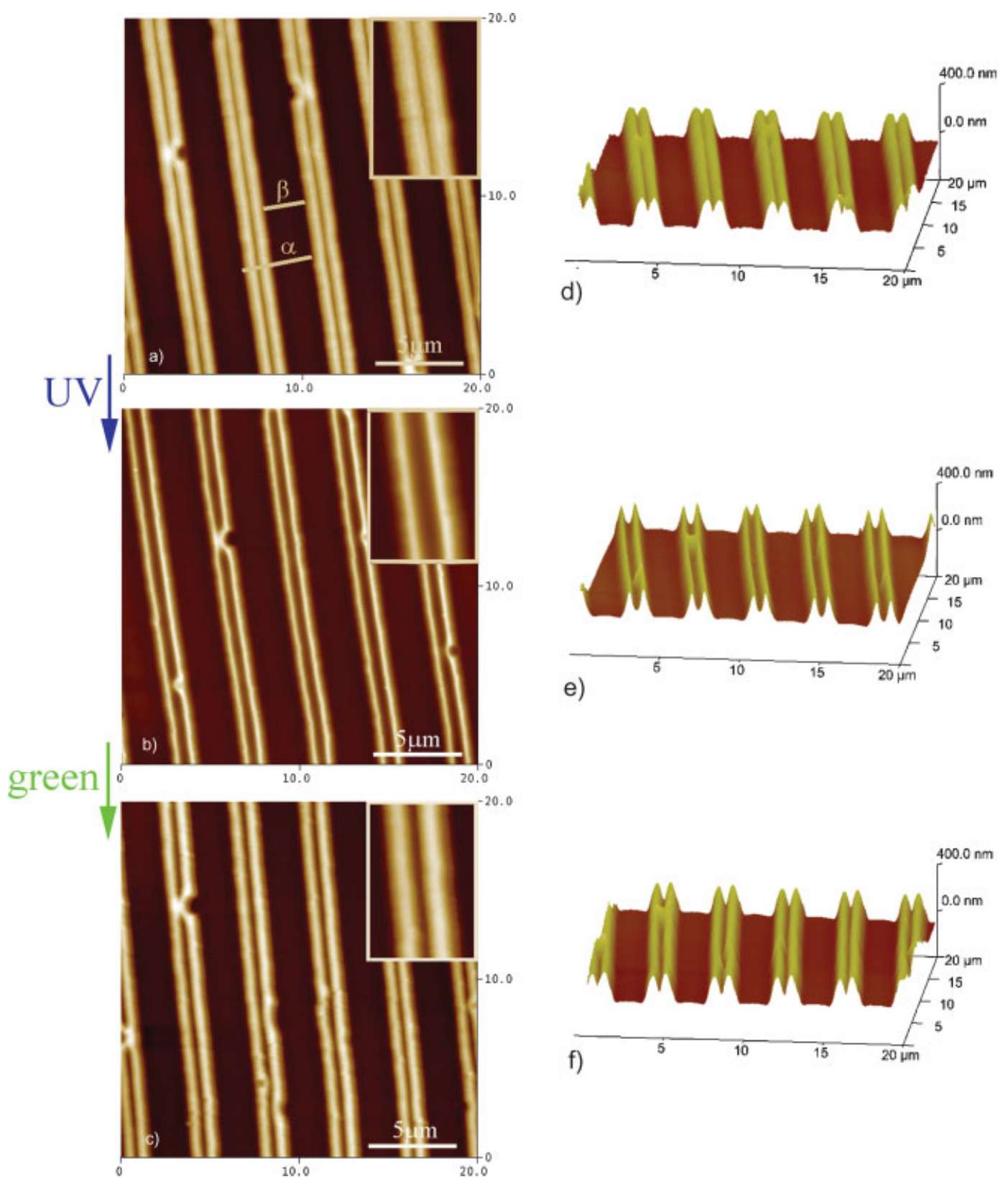

Figure 5. AFM images of a grating a) before any irradiation where $\alpha$ is the period and $\beta$ is the distance between the stripes, measured at the full width at half-maximum of each feature, b) after irradiation with 20 UV pulses, and c) after subsequent exposure to 600 green pulses. The insets demonstrate a single stripe in magnification. $d-f$ ) 3D images of the grating shown in $(a-c)$, respectively. The images demonstrate the optically switchable structural changes of the grating.

ratio $I_{1} / I_{0}$, and consequently the DE for the gratings before and after the UV-visible irradiation cycle.

The theoretical calculations confirm the increase of the DE after the photoisomerization of SP to MC. Particularly, the relative change of the DE predicted by the above theory after the SP-to-MC transformation was $8.7 \%$. The agreement between the calculated and experimental (ca. $7.4 \pm 3.0 \%$ ) values is remarkable, taking into account the experimental error due to factors such as the exact value of the spot size of the laser beam, which leads to an approximate value of the observed stripes, the imperfections of the surface introduced during the grating formation, and so on.

In the majority of other studies, where thin gratings in the Raman-Nath regime are produced, azobenzene polymers and liquid crystals are used. In these studies the gratings are formed after irradiation with interfering beams that cause variation of the refractive index inside the sample, and the switching procedure in the DE is because of the formation and deletion of the gratings. Yamamoto et al. ${ }^{[16]}$ produced this kind of gratings, with a thickness of about $500 \mathrm{~nm}$ and a period of $2 \mu \mathrm{m}$, 
close to the characteristics of our gratings. In contrast to our experiments that take place under ambient conditions, their DE switching was performed under high temperatures of approximately $110-143^{\circ} \mathrm{C}$. Initially no grating was present on the samples, and at the temperature closer to the ambient one $\left(110^{\circ} \mathrm{C}\right)$ the maximum first-order diffraction efficiency of the formed gratings was $0.44 \%$. Fu et al. ${ }^{[12]}$ used polymer films containing spirooxazine compounds to perform holographic gratings with thicknesses of $10 \mu \mathrm{m}$, based on the refractiveindex variation in the samples. They also start without a grating and the maximum DE of the formed grating after irradiation with interfering light is ca. $0.1 \%$. On the other hand, Tong et al. ${ }^{[15]}$ used azobenzene-polymer-stabilized liquid crystals for dual-mode switching of DE, and showed the possibility of modification of the DE of an already formed grating with large relative changes. For gratings with a period comparable to the gratings presented here $(5 \mu \mathrm{m})$, but with a thickness two orders of magnitude higher, the relative DE changes were about $50 \%$ and the initial DE is one order of magnitude lower than the one measured in our gratings. It should be noticed here that although the thickness of our gratings is very small, they exhibit higher DE than most of the volume gratings prepared in this regime. From our theoretical calculations, it is clear that the decrease of the dimensions of the stripes of the gratings and of the period, which leads to the increase in the number of the observed stripes, are the main parameters that define the change in the DE of the prepared gratings upon irradiation. For this reason, experiments are on their way that focus on the improvement of the DE changes by modifying the substrate characteristics (concentration, different kind of dopants, different matrix) or the rating characteristics (duty cycle, period) that can affect the above parameters.

\section{Conclusions}

This work demostrates the possibilty of manipulating the diffraction efficiency of thin gratings in a desired way. Specifically, we produced gratings on a layer of polymer that is mixed with photochromic SP molecules, that change their diffraction efficiency in a reversible way upon laser irradiation. This effect is attributed to the reversible dimensional changes of the imprinted structures, and not to the refractive index changes as is the case in the majority of previous work. The experimental results are confirmed by a theoretical diffraction model. The findings open a way for the production of optically switchable gratings based on reversible dimensional changes, and can be of great importance in all-optic signal processing systems.

\section{Experimental}

Sample Preparation: For the preparation of the films, solutions of $90.0 \mathrm{wt} \%$ of the polymer polyethylmethacrylate-co-methylacrylate (PEMMA) (average molecular weight, $M_{\mathrm{w}} \sim 100,000$ ) (Aldrich) and
$10.0 \mathrm{wt} \%$ of the photochromic molecule $1^{\prime}, 3^{\prime}$-dihydro- $1^{\prime}, 3^{\prime}, 3^{\prime}-$ trimethyl-6-nitrospiro[2H-1-benzopyran-2,2'-(2H)-indole] or $\left(6-\mathrm{NO}_{2}\right.$ BIPS) (Aldrich) were prepared in toluene. A certain volume of this solution was spin-coated onto a glass substrate $(300 \mathrm{rpm}$ for $1 \mathrm{~s}$ following $1000 \mathrm{rpm}$ for $30 \mathrm{~s}$ ).

Grating Preparation: The resulting substrates were placed on a hot plate and heated until they reach a temperature $T_{\text {grating }}$ of $65^{\circ} \mathrm{C}$. Then an elastomeric mold (period $\alpha=4 \mu \mathrm{m}$ ) was placed on the substrate for $10 \mathrm{~min}$, resulting in the formation of the gratings. This procedure took place in a controlled nitrogen atmosphere. In this study, for the formation of the gratings we used the soft-molding technique [18], which is based on the conformal contact between the material to be patterned and an elastomeric replica of a master structure. It combines soft and nanoimprint lithography, using elastomeric elements and exploiting the glass transition of organic compounds.

Experimental Setup: The irradiation cycles were made by a Nd:YAG laser operating at the second and third harmonic, $\lambda_{1 \text { laser }}=$ $532 \mathrm{~nm}$ and $\lambda_{\text {2laser }}=355 \mathrm{~nm}, \tau_{\text {pulse }} \approx 5-6 \mathrm{~ns}$ (Spectra Physics). The laser beams reach the front surface of the film, so that the entire area of the grating is homogeneously irradiated (spot area: ca. $19 \mathrm{~mm}^{2}$ for UV and ca. $12 \mathrm{~mm}^{2}$ for green). The fluences of UV- and green-laser light used for the irradiation were $F_{355}=20 \mathrm{~mJ} \mathrm{~cm}^{-2}$ and $F_{532}=35 \mathrm{~mJ} \mathrm{~cm}^{-2}$, respectively.

For the measurement of the DE, a continuous-wavelength diode laser, operating at $\lambda_{\text {laser }}=822 \mathrm{~nm}$ with spot diameter of $1 \mathrm{~mm}$ and energy much lower than $1 \mathrm{~mW}$, was used (reading beam). The zero-order (transmitted, $I_{0}$ ) and first-order diffracted beam $\left(I_{1}\right)$ were simultaneously recorded by two identical photodiodes connected with a lock-in amplifier system. The active area of the two photodiodes was much bigger than the diameter of the transmitted and first diffracted beam, and in each measurement we made sure that the beams were always inside this active area. The lock-in based measurement system constitutes a light chopper with chopping frequency of $379 \mathrm{~Hz}$ (OC-4000 Photon Technology International), a lock-in amplifier (SR830-DSP Stanford Research Systems), and a computer. For each sample we took sequential measurements after irradiation with $0,5,10$, $20 \mathrm{UV}$ pulses and 50,100, up to 600 every 100 green pulses in each cycle. Each measurement consists of 200 points and for each point the integration time was $100 \mathrm{~ms}$. From the raw data recorded, the mean value of $I_{1}$ or $I_{0}$ in each case was derived and used for further analysis. The irradiation cycles and the measurements of the DE were performed at room temperature.

Absorption Measurements: UV-visible absorption spectra were obtained using a UV-vis-NIR spectrophotometer (Cary 5000-Varian) on PEMMA-SP samples on glass.

Ellipsometric Measurements: The ellipsometric measurements were performed by a spectroscopic ellipsometer (MM-16 Horiba Jobin Yvon) at an angle of incidence of $70^{\circ}$ across the spectral range $430-850 \mathrm{~nm}$. The index of refraction was calculated according to a single-layer model. The measurements were performed at room temperature.

AFM Measurements: The AFM experiments were performed with a Nanoscope III microscope (Digital Instruments) equipped with a $15 \mu \mathrm{m}$ scanner and operating by tapping mode in air. We used phosphorous-doped Si tips with a $10 \mathrm{~nm}$ nominal curvature radius and a scanning rate of $0.5 \mathrm{~Hz}$.

Microscopy Measurements: The microscopy measurements were performed with a reflected-light bright-field microscope (Leica DM2500 M).

DSC Measurements: Thermal analysis was carried out using a Perkin-Elmer Pyris Diamond Intracooler Ready differential scanning calorimeter. The instrument was previously calibrated with the indium standard. Runs were carried out with automatic baseline correction, and the baseline was recorded by a preliminary scan from -50 up to $350{ }^{\circ} \mathrm{C}$ with a rate of $10^{\circ} \mathrm{Cmin}^{-1}$. For the measurements, about $9 \mathrm{mg}$ of material was put in standard aluminum pans (Perkin-Elmer); after drying and re-humidifying, two consecutive scans over a range of 
temperature from -30 to $250{ }^{\circ} \mathrm{C}$ with a rate of $10^{\circ} \mathrm{Cmin}^{-1}$ were performed. The glass-transition temperature of the samples was evaluated from the second scan with the Half-Cp extrapolated method.

Received: October 30, 2007

Revised: January 23, 2008

Published online: May 21, 2008

[1] A. Shacham, B. A. Small, O. Liboiron-Ladouceur, K. Bergman, $J$. Lightwave Technol. 2005, 23, 3066.

[2] K. A. Crandall, M. R. Fisch, R. G. Petschek, C. Rosenblatt, Appl. Phys. Lett. 1994, 64, 1741.

[3] J.-N. Kuo, H.-W. Wu, G.-B. Lee, Opt. Express 2006, 14, 6844.

[4] B. H. Soffer, J. D. Margerum, A. M. Lackner, D. Boswell, A. R. Tanguay, Jr., T. C. Strand, A. A. Sawchuk, P. Chavel, Mol. Cryst. Liq. Cryst. 1981, 70, 145

[5] C. Gu, Y. Xu, Y. Liu, J. J. Pan, F. Zhou, H. He, Opt. Mater. 2003, 23 , 219.

[6] Y. Huang, W. Liang, J. K. S. Poon, Y. Xu, R. K. Lee, A. Yariv, Appl. Phys. Lett. 2006, 88, 181102.

[7] a) R. L. Sutherland, V. P. Tondiglia, L. V. Natarajan, T. J. Bunning, W W. Adams, Appl. Phys. Lett. 1994, 64, 1074. b) Y. Zhao, X. Tong, $A d v$. Mater. 2003, 15, 1431.

[8] P.-T. Lin, X. Liang, H. Ren, S.-T. Wu, Appl. Phys. Lett. 2004, 85, 1131.

[9] A. K. Ghosh, Y. Takanishi, K. Ishikawa, Y. Ono, J. Kawamura, H. Takezoe, J. Appl. Phys. 2004, 95, 5241.

[10] A. K. Ghosh, S.-W. Choi, Y. Takanishi, B. Park, K. Ishikawa, Y. Ono, J. Kawamura, H. Takezoe, J. Appl. Phys. 2004, 96, 5909.

[11] F. Gallego-Gomez, M. Salvador, S. Köber, K. Meerholz, Appl. Phys. Lett. 2007, 90, 251113.

[12] S. Fu, Y. Liu, Z. Lu, L. Dong, Z. Lu, W. Hu, M. Xie, Opt. Mater. 2005 27, 1567.

[13] a) M. Ishiguro, D. Sato, A. Shishido, T. Ikeda, Langmuir 2007, 23, 332. b) J. Kumar, L. Li, X. L. Jiang, D.-Y. Kim, T. S. Lee, S. Tripathy, Appl. Phys. Lett. 1998, 72, 2096.

[14] N. Zettsu, T. Ogasawara, R. Arakawa, S. Nagano, T. Ubukata, T. Seki, Macromolecules 2007, 40, 4607.
[15] X. Tong, G. Wang, A. Yavrian, T. Galstian, Y. Zhao, Adv. Mater. $\mathbf{2 0 0 5}, 17,370$

[16] T. Yamamoto, A. Ohashi, S. Yoneyama, M. Hasegawa, O. Tsutsumi, A. Kanazawa, T. Shiono, T. Ikeda, J. Phys. Chem. B 2001, 105, 2308.

[17] A. Saishoji, D. Sato, A. Shishido, T. Ikeda, Langmuir 2007, 23, 320.

[18] D. Pisignano, L. Persano, R. Cingolani, G. Gigli, F. Babudri, G. M. Farinola, F. Naso, Appl. Phys. Lett. 2004, 84, 1365.

[19] A. Athanassiou, M. Kalyva, K. Lakiotaki, S. Georgiou, C. Fotakis, Adv. Mater. 2005, 17, 988.

[20] A. Athanassiou, K. Lakiotaki, V. Tornari, S. Georgiou, C. Fotakis, Appl. Phys. A 2003, 76, 97.

[21] a) G. Smets, $A d v$. Polym. Sci. 1983, 50,17. b) A. Tork, F. Boudreault, M. Roberge, A. M. Ritcey, R. A. Lessard, T. V. Galstian, Appl. Opt. 2001, 40, 1180. c) H. Bouas-Laurent, H. Durr, Pure Appl. Chem. 2001, 73,639 .

[22] N. Tamaoki, E. Van Keuren, H. Matsuda, K. Hasegawa, T. Yamaoka, Appl. Phys. Lett. 1996, 69, 1188.

[23] H. S. Blair, H. I. Pogue, Polymer 1982, 23, 779.

[24] S. Meng, T. Kyu, L. V. Natarajan, V. P. Tondiglia, R. L. Sutherland, T. J. Bunning, Macromolecules, 2005, 38, 4844.

[25] S. Krause, Pure Appl. Chem. 1986, 58, 1553.

[26] a) T. Masubuchi, H. Fukumura, H. Masuhara, K. Suzuki, N. Hayashi, J. Photochem. Photobiol. A 2001, 145, 215. b) T. Masubuchi, H. Furutani, H. Fukumura, H. Masuhara, J. Phys. Chem. B 2001, 105 , 2518.

[27] G.-D. Liang, J.-T. Xu, Z.-Q. Fan, S.-M. Mai, A. J. Ryan, Langmuir 2007, 23, 3673.

[28] A. Athanassiou, D. Sahinidou, V. Arima, S. Georgiou, R. Cingolani, C. Fotakis, J. Photochem. Photobiol. A 2006, 183, 182.

[29] S. Zankovych, T. Hoffmann, J. Seekamp, J.-U. Bruch, C. M. Sotomayor Torres, Nanotechnology 2001, 12, 91.

[30] P. S. Hong, H. H. Lee, Appl. Phys. Lett. 2003, 83, 2441.

[31] K. Sasaki, T. Nagamura, J. Appl. Phys. 1998, 83, 2894.

[32] M. Born, E. Wolf, Principles of Optics: Electromagnetic Theory of Propagation, Interference and Diffraction of Light, 7th Edition, Cambridge University Press, Cambridge, UK 1999. 